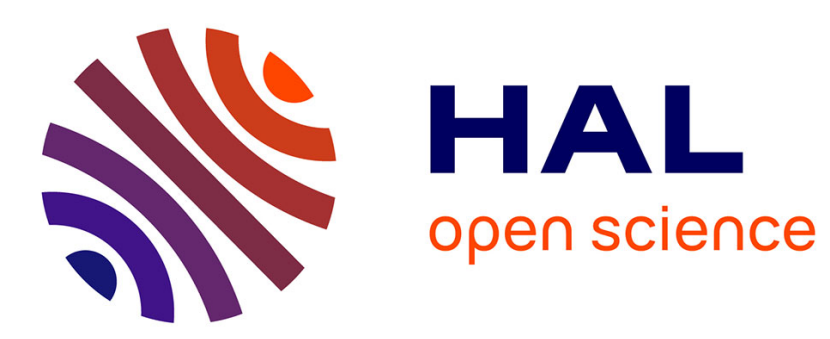

\title{
Amour de l'adrénaline et de la nature
}

Gilles Raveneau, Géraldine Fasnacht

\section{To cite this version:}

Gilles Raveneau, Géraldine Fasnacht. Amour de l'adrénaline et de la nature. Ethnologie française, 2006, Les sports à risques, 36 (4), p. 687-689. 10.3917/ethn.064.0687 . hal-03135207

\section{HAL Id: hal-03135207 https://hal.parisnanterre.fr/hal-03135207}

Submitted on 8 Feb 2021

HAL is a multi-disciplinary open access archive for the deposit and dissemination of scientific research documents, whether they are published or not. The documents may come from teaching and research institutions in France or abroad, or from public or private research centers.
L'archive ouverte pluridisciplinaire HAL, est destinée au dépôt et à la diffusion de documents scientifiques de niveau recherche, publiés ou non, émanant des établissements d'enseignement et de recherche français ou étrangers, des laboratoires publics ou privés. 


\section{Amour de l'adrénaline et de la nature}

Propos recueillis par Gilles Raveneau

Géraldine Fasnacht

snowboardeuse

Géraldine Fasnacht est née le 18 juin 1980 à Lausanne. Elle habite en Suisse dans le Val de Bagne, près de Verbier. Elle a commencé à faire du snowboard à l'âge de dix ans avec sa mère, qui souhaitait essayer cette nouvelle pratique. À quinze ans, elle participe à ses premières compétitions en freestyle ${ }^{1}$. Puis elle se dirige progressivement vers le freeride ${ }^{2}$, auquel elle se consacre depuis. Ses études de commerce terminées et après des séjours à l'étranger pour perfectionner ses langues étrangères (allemand, anglais et espagnol), elle travaille chez Swissair durant plusieurs années, puis comme agent de voyages chez Verbier Tours. En 1999, tout en continuant à se vouer au snowboard, elle commence à faire du parachutisme. Après trois cents sauts d'avion, elle s'élance de sa première falaise en BASE-jump.

Semi-professionnelle dans sa discipline, Géraldine Fasnacht développe parallèlement, avec le guide de montagne Sébastien Gay, la société de production Line Prod, qui crée des vidéos et des événements autour des sports extrêmes. La snowboardeuse organise par ailleurs des stages de freeride et anime une école de montagne pour enfants. De 1997 à 1999, elle prend la première place de différentes compétitions de freestyle (à Verbier, à Morgin, Thun et aux Diablerets). Puis, elle excelle rapidement en freeride. En 2002, elle est première aux Arcs freeride, au Chandolin freeride, à l'X-treme de Verbier et au Cannibal Thyon freestyle. En 2003, elle remporte le prix Gilles Voirol à l'X-treme de Verbier. En 2004 et 2006, elle prend la première place de l'Engadinsnow.com freeride international, et la seconde de l'X-treme de Verbier en 2004 et 2005. L'X-treme de Verbier est la plus importante compétition au monde de freeride extrême : une face de 800 mètres à $55^{\circ}$ d'inclinaison, sur laquelle les concurrents affrontent des falaises de 20 à 25 mètres.

Le snowboard est vital pour moi. J'en ai besoin. Tous les moments passés en montagne m'apportent énormément. Cela me gorge d'énergie et me vide l'esprit de tous les soucis et les bêtises du quotidien. J'ai toujours l'impression d'y découvrir quelque chose. Je ne saurai jamais tout sur la montagne, contrairement à mon travail précédent. Quand je n'apprends plus rien, cela m'énerve et je veux passer à autre chose. En montagne, je n'ai jamais eu ce sentiment. Il y a cet échange avec la nature qui me remplit.

Je n'ai pas l'impression de me mettre en danger, à partir du moment où je connais mes limites et les respecte. Il y a des choses que je ne me permets pas. J'ai toujours des peurs. Celui qui dit qu'il n'a pas peur en montagne, il doit se remettre en question parce que c'est là qu'il aura un accident mortel. La peur est importante. Elle permet de connaître où sont ses limites. Mais le jour où on est effrayé de tout ce qu'on fait en montagne, il faut arrêter. Si on est trop sûr de soi, on prend les choses à la légère et c'est là que l'accident arrive. Il faut être lucide et ne jamais se dire qu'on en fait trop parce que c'est ce jour-là qu'on recevra la leçon. Il faut rester humble. À côté d'une montagne, on est une puce au milieu de l'océan.

Je ne pense pas prendre plus de risques dans mon activité sportive que dans la vie de tous les jours. Lorsque je vivais comme tout le monde et que je travaillais à Swissair, j'empruntais chaque jour la route entre Lausanne et Genève-aéroport. J'ai failli m'y tuer plus souvent qu'en montagne! Aux heures de pointe et avec les gens qui conduisent n'importe comment ! Et cela sans compter le stress de la vie quotidienne, les agressions en ville, tout ce genre de choses. Là, je vis une vie saine. Je suis au milieu des montagnes, près de la nature, avec des gens authentiques et passionnés. Je vis mon rêve. Ce n'est pas plus dangereux que de vivre en ville tous les jours et de travailler comme un fou jusqu'à n'en plus finir. J'ai vraiment l'impression d'être dans mon élément ici.

Je ne dis pas que je peux tout contrôler en montagne, mais je l'admets. Cela ne signifie pas que j'accepte de mourir à n'importe quel moment. Dans tous les cas, je préfere mourir en montagne que sur la route. Je sais aussi que je ne connaitrai jamais assez la montagne pour

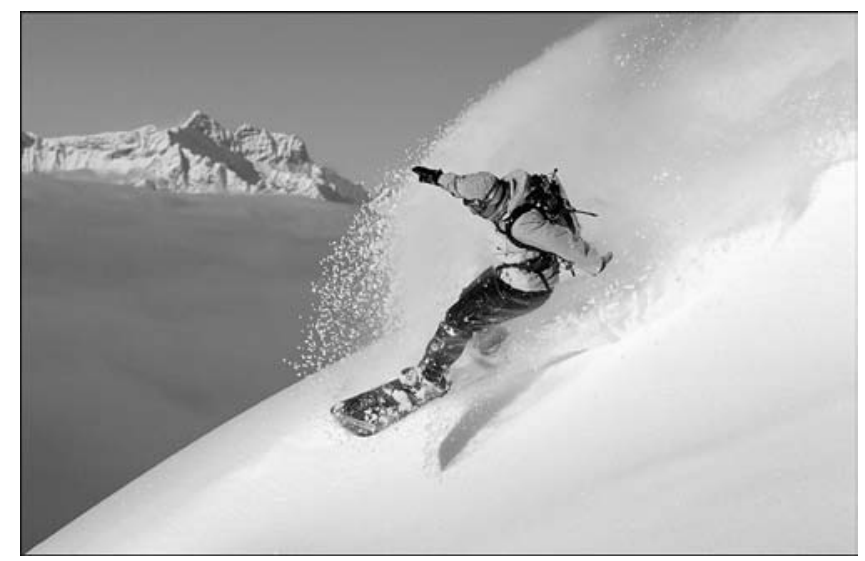

1. 2. 3. Géraldine Fasnacht photographiée par Mark Shapiro. 


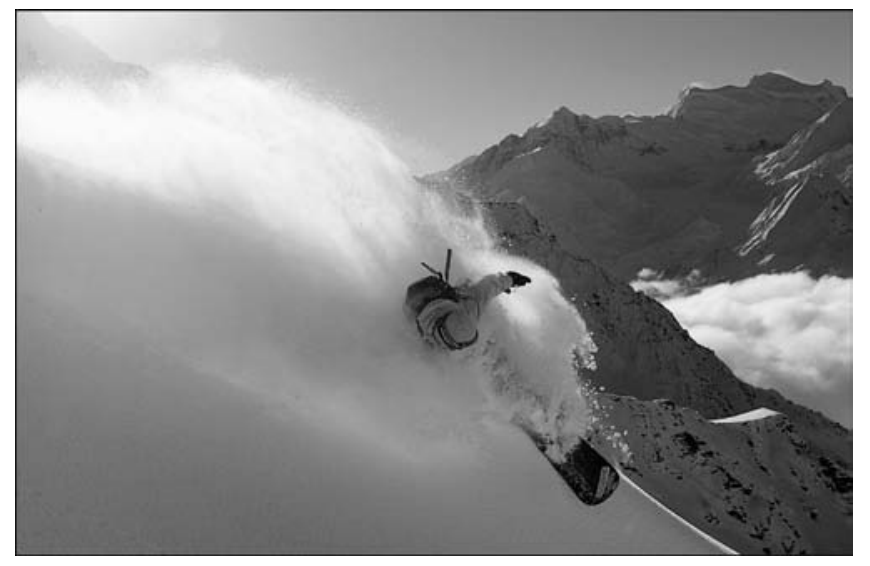

savoir tout ce qu'elle cache. J'essaie de prendre un maximum de précautions, d'y aller avec des gens de confiance et de mettre toutes les chances de mon côté. Mais on ne peut pas tout prévoir, surtout quand le milieu naturel se déchaîne.

Il faut aussi faire confiance à notre petite voix intérieure lorsqu'on sent qu'il ne faut pas y aller. Il m'arrive de ne pas être à l'aise, d'avoir un mauvais pressentiment, aussi bien en BASE-jump sur le haut d'une falaise qu'au sommet d'une face avant de descendre en freeride. Il reste plein d'autres journées. Ce n'est pas parce qu'on rate un "ride " et qu'il faut redescendre à pied pendant quatre heures que c'est grave. On a fait une jolie balade et puis voilà. L'une de ces expériences-là se déroulait en Afrique. Nous avions grimpé, Sébastien Gay et moi, une falaise pendant huit heures avec nos parachutes sur le dos. En arrivant au sommet, le vent était trop fort pour sauter. Nous décidons alors de dormir au sommet de la falaise et d'attendre le lendemain matin. Au petit matin, le vent n'avait pas faibli et nous n'avions pratiquement plus rien à manger et à boire. Nous sommes alors redescendus en rappel pendant quatre heures jusqu'au camp. On est remontés le surlendemain et là on a pu sauter.

C'est identique en freeride. Je me souviens d'une fois où j'étais au sommet d'une face avec trois copains qui me disaient: "Allez, viens! les conditions sont superbes!" Mais je ne le sentais pas. Ils sont partis face nord, moi je suis redescendue de l'autre côté. Je dois "rider" l'esprit libre, sinon je n'aurai pas de plaisir. En montagne, il y a toute une série de petits indices difficiles à percevoir: une plaque qui part dans la montée, le gros "boum» des commencements d'avalanches prêtes à décrocher, le sommet de la corniche de départ qui s'effondre lorsqu'on saute dessus... Là, on se dit : "Je n'y vais pas. " Le plus grand risque est de ne pas savoir dire non. Ce n'est pas nous qui décidons, mais la nature. Il faut savoir l'écouter. Si elle n'a pas envie de nous, elle le fait vite comprendre.

Je ne crois pas avoir le goût du risque, mais plutôt l'amour de l'adrénaline, des sensations et surtout de la

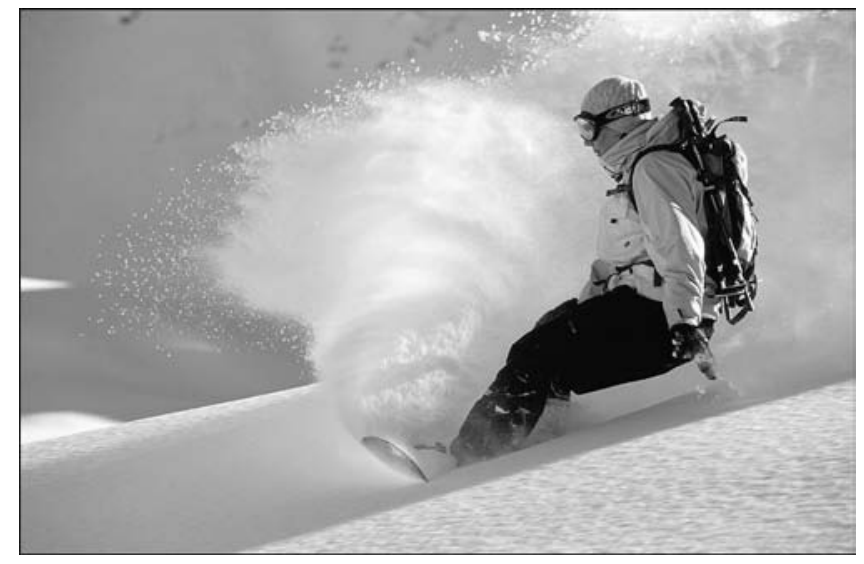

nature. J'ai besoin de l'adrénaline. C'est comme une drogue, même si je ne me suis jamais droguée. C'est une sorte de dépendance par rapport à ces sensations, à ces moments où l'on descend dans une pente fabuleuse de poudreuse, où l'on a l'impression de voler sur sa planche comme sur une vague en mouvement. Ce sont des sensations tellement courtes et intenses que j'ai besoin de les renouveler. J'ai l'impression de faire corps avec la nature et d'être en osmose avec elle, de partager quelque chose de vrai, comme si j'arrivais à lui parler. Quand je regarde voler un oiseau en quittant une falaise par exemple, les gestes qui me viennent à l'esprit sont les mêmes qu'eux. Lorsque je traverse à toute allure un couloir en snowboard, en volant au-dessus de la poudreuse et en sautant une barre de rochers, j'ai des visions fugaces que je n'ai jamais autrement. J'ai le sentiment d'avoir une autre clairvoyance, d'être dans une autre dimension. Je ne le retrouve pas ailleurs.

L'adrénaline provoque en moi une sorte d'euphorie. Un mélange de plénitude, de frissons et de joie. Quand j'arrive en bas, j'ai tout le corps qui tremble, tellement c'était bon. C'est une sensation que j'ai besoin de ressentir régulièrement. Hier par exemple, je me suis fait une journée de "ride» qui était incroyable. À chaque fin de "run", c'était simplement le nirvana. C'était génial. Pendant la descente, on est tellement confiant et concentré. On sait exactement où passer : quel rocher éviter ou sauter, s'engager à gauche ou à droite, quel couloir prendre. On sait pratiquement que la neige va couler à cet endroit et qu'ici elle partira, mais qu'on ira plus vite qu'elle. On a une ligne définie dans la tête. On doit la suivre. Ça doit se dérouler comme prévu. Il existe bien des échappatoires, mais leur nombre est très limité sur une pente. Une erreur peut conduire à se blesser gravement ou à y rester. Le mental est essentiel, la concentration doit être complète.

C'est ma vie, je n'ai pas envie de jouer avec. Ce n'est pas un jeu, je le prends au sérieux. Je suis tellement concentrée et ma ligne est si bien définie. Parler de jeu signifierait qu'on pourrait rejouer si cela se passait mal. 
Or, dans des pentes très engagées, on ne peut pas. Sur des faces moins dangereuses où la chute est sans conséquence, là d'accord.

J'ai envie de vivre à fond tous ces sports extrêmes et ces moments dans la nature. Je ne désire pas une vie de famille ou un enfant pour le moment, mais cela m'arrivera peut-être. Les copines qui partagent ce sport sont dans le même trip que moi. En général, nous sommes bien acceptées par les garçons, vu que nous ne sommes déjà pas nombreuses. Il y a même souvent de la fierté d'avoir une fille dans l'équipe. Il n'y a pas de différence du moment que l'on suit. Ce n'est pas parce que je suis une femme que je vais le vivre différemment d'un homme. Et ce n'est pas parce j'ai un corps qui est fait pour avoir des enfants que je ne peux pas faire ce genre de sport.

\section{Notes}

1. Il s'agit d'une modalité de pratique où le concurrent cherche à faire des sauts et à privilégier les figures en l'air et le style plutôt que la vitesse.

2. C'est le hors-piste dans la neige pou- dreuse, sur de belles façades montagneuses permettant de jouer avec la pente en de grandes et belles courbes pour tracer la ligne la plus pure. 